\title{
ILLUSORY MOTION IN DEPTH: AFTEREFFECT OF ADAPTATION TO CHANGING SIZE ${ }^{1}$
}

\author{
D. Regan and K. I. BEVERLEY \\ Department of Psychology, Dalhousie University, Halifax, Nova Scotia, Canada B3H 4J1
}

(Received 20 March 1977)

\begin{abstract}
After adapting to changing size by viewing a square whose dimensions increased with a ramp waveform, a subsequently-viewed test square appeared to move continuously away in depth. Adapting to decreasing size produced the opposite aftereffect. This depth movement aftereffect could be measured by cancelling it by some unique rate of change of size. The direction of the aftereffect and the direction of the cancelling stimulus were independent of whether the adapting square of test square was of positive or negative contrast. The aftereffect built up over 10 min adaptation and decayed exponentially $(\tau=52 \mathrm{sec}$ ). It cannot be explained in terms of the classical movement aftereffect. We propose that neural filters sensitive to unidirectionally changing size drive the neural mechanism that underlies the perception of motion in depth.
\end{abstract}

Key Words-motion; aftereffect; size; stereoscopic depth movement.

\section{INTRODUCTION}

We have reported psychophysical evidence for the existence of information-processing channels sensitive to the relative velocities of the left and right retinal images, and thereby tuned to different directions of motion in three dimensions. These channels are not accessible to monocular stimulation and constitute a stereoscopic system for motion that is quite distinct from the classical stereoscopic system for position. They feed the neural organization that underlies the perception of motion in depth (Beverley and Regan, 1973a,b; 1975). Microelectrode recording in area 18 of cat visual cortex has revealed neurons tuned to the direction of motion in depth whose properties provide a physiological basis for these cyclopean channels (Cynader and Regan, 1978).

Here we report experimental support for the proposal that there exists a second input to the neural organization that underlies the perception of motion in depth. This input is provided by neural filters that can be driven either monoculariy or binocularly by changing size.

\section{METHODS}

The stimulus was a bright square with a mean side length of $1^{\circ}$ and luminance $27 \mathrm{~cd} / \mathrm{m}^{2}$. The square was generated on a Tektronix type 604 CRO with a green type 31 phosphor and was optically superposed on a white $15^{\circ} \times 10^{\circ}$ adapting background of luminance $4.3 \mathrm{~cd} / \mathrm{m}^{2}$. The viewing distance was $145 \mathrm{~cm}$. The subject's head was supported in an adjustable rest.

Adapting and test stimuli were ramping changes of size. There was always an $0.25 \mathrm{sec}$ blanking interval between successive ramps. Settings were made by the method of adjustments. For the Fig. 1 data the adaptation period was set at $20 \mathrm{~min}$, since prior experiment showed that a longer period produced negligibly more effect. Settings were made within $15 \mathrm{sec}$ with 1 min readaptation between settings. For the data of Fig. 2 the adapting stimulus was

${ }^{1}$ This work was presented at the April 1977 ARVO Meeting, Sarasota. a rate of size change of $24 \mathrm{~min}$ arc/sec with a ramp time of $1.0 \mathrm{sec}$. The test ramp time was also $1.0 \mathrm{sec}$. Each setting to null the aftereffect was made within $5 \mathrm{sec}$. Other experimental details were as for the Fig. 1 experiment.

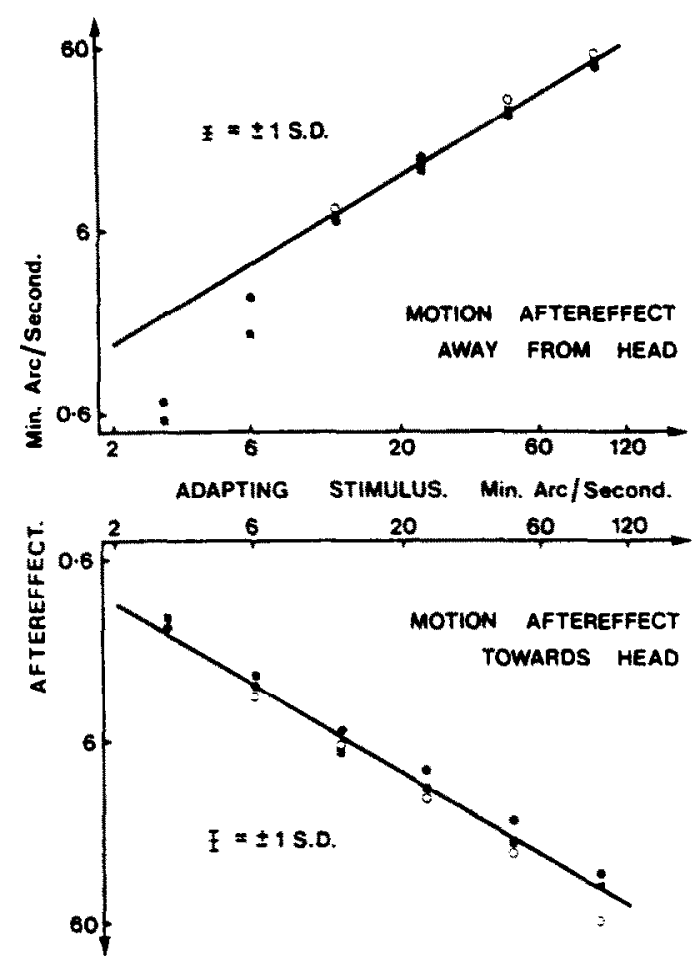

Fig. 1. Rates of size change required to null motion in depth aftereffect versus the adapting rates of size change (both axes log min arc/sec). Upper adaptation to increasing size-motion aftereffect away from head: Lower adaptation to decreasing size-motion aftereffect towards head. Adapting ramp time was $1.0 \mathrm{sec}$ with a $0.25 \mathrm{sec}$ blanking interval between successive ramps. Three test ramp times of 0.25 (open circles), 1.0 (filled squares) and $3.3 \mathrm{sec}$ (filled circles) all with $0.25 \mathrm{sec}$ blanking intervals were used. The mean \pm 1 standard deviation for all points is shown: all SDs were similar on log axes. Subject K.B. 

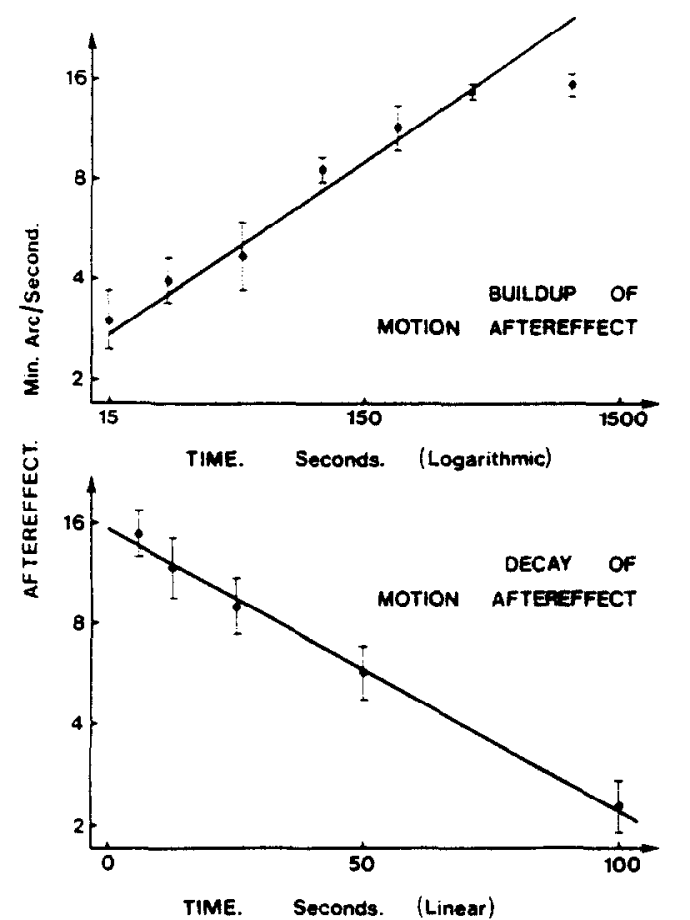

Fig. 2. Buildup (upper) and decay (lower) of the motion aftereffect. The ordinates plot the rate of change of size required to null the motion-in-depth aftereffect $(\log \mathrm{min}$ arc/sec). The abscissa for the buildup is the duration of adaptation (log sec): the abscissa for the decay is the interval between the cessation of $(20 \mathrm{~min})$ adaptation and the measurement of the aftereffect (sec). The vertical lines show I I standard deviation: all SDs were similar on log axes.

In experiments dealing with positive versus negative contrasts we used a white adapting background $\left(10^{\circ} \times 15^{\circ}\right)$ of luminance $6.4 \mathrm{~cd} / \mathrm{m}^{2}$. Superposed on this was a green patch $\left(5^{\circ} \times 4^{\circ}\right)$ of luminance $2.7 \mathrm{~cd} / \mathrm{m}^{2}$ in the middle of which was a $1^{3} \times 1^{2}$ bright square of luminance $5.4 \mathrm{~cd} / \mathrm{m}^{2}$ (approximately $30 \%$ positive contrast) or a $1^{\circ} \times 1^{\circ}$ dark square of luminance $0 \mathrm{~cd} / \mathrm{m}^{2}$ (approximately $30 \%$ negative contrast).

Two subjects were used (the authors), both experienced in psychophysical experiments.

\section{RESULTS}

Motion-in-depth aftereffect and nulling of aftereffect

Using his right eye a subject gazed steadily for 20 min at a dark fixation point in the centre of the bright square while the square's side length increased at a fixed rate (e.g., $24 \mathrm{~min} \mathrm{arc} / \mathrm{sec}$ ) with a ramp time of $1.0 \mathrm{sec}$. During the ramp changes of square size the square appeared to be moving in depth. We report that both subjects noted a compelling negative movement aftereffect: the subsequently-viewed static test square appeared to be moving continuously away from the head along the line of sight. Conversely, after adapting to decreasing size, a static test square appeared to move continuously towards the head. (As with the classical movement aftereffect, the illusory motion was not accompanied by changing location, the necessary accompaniment of real motion.)

We attempted to null this illusory motion in depth by causing the test square to continuously change size in the same sense as the adapting stimulus. It did indeed prove possible to null the aftereffect in this way. The rate of change of size required to null the illusory depth motion provided a quantitative measure of the aftereffect

Figure 1 shows the relation between the strength of the adapting stimulus and the size of the aftereffect as measured by nulling. Over a wide range the relation was approximately linear on $\log -\log$ axes. Thus for all but the weakest adapting stimulus the aftereffect obeyed the equation $V_{v}=k V_{A}^{n}$ where $V_{y}$ was the rate of size change to null the aftereffect, $l_{+}$was the adapting rate of size change, and $k$ and $n$ were constants (rates of change of side length are in min arc sec). For subject K.B., constant $n$ was equal to 0.9 ? and 0.94 for adaptation to increasing and decreasing size respectively. So that approximately $V_{\mathrm{y}}=k V_{4}$ Constant $k$ was equal to 0.77 for increasing size and 0.56 for decreasing size. For subject D.R. the corresponding values for $n$ were 0.86 and 0.70 . and for $k$ were 0.52 and 0.84 . The near-unity values for $n$ were in accord with the qualitative subjective impression that the speed at which a static test target appeared to move in depth was roughly proportional to the rate of change of size of the prior adapting stimulus. Only for weak adapting stimuli whose rate of size change was less than about $6 \mathrm{~min}$ arc/sec was there any evidence for a threshold effect. For the weakest adapting stimuli no aftereffect was visible using the $0.25 \mathrm{sec}$ test ramp, possibly due to the greater difficulty in making the measurement with the short ramp times.

If the effective parameter of the nulling stimulus were excursion distance rather than the rate of size change, then our data could be explained in a differ. ent and less interesting way. Since all our stimuli were repetitive, rate of change of size was confounded with excursion distance. In order to disentangle the effects of these two parameters we adopted a "factorial" design and tested with three different ramp durations. Thus, each nulling rate of size change corresponded to three different excursions. We plotted the data of Fig. 1 in two ways, namely with ordinates in units of distance (excursion) and also with ordinates in terms of rate of size change (as shown). Only when ordinates were in terms of rate of size change did data points for the three ramp durations coincide (Fig. 1), showing that a given aftereffect was nulled by the same rate of size change independently of test stimulus excursion. Therefore, the effective nulling parameter was rate of size change rather than excursion.

\section{Buildup and decay of motion-in-depth aftereffect}

The time courses of buildup and decay for the depth motion aftereffect are shown in Fig. 2. Buildup (upper) was continuous up to $10 \mathrm{~min}$ at least. Figure 2 (upper) shows that, when the buildup was plotted on $\log -\log$ axes, except for the longest adapting time (20 $\mathrm{min})$, all points were a reasonable fit to a straight line of slope 0.48 . Therefore, buildup obeyed the equation $V_{N}=k t^{0.48}$. This power function approximates the square root law $V_{\mathrm{v}}=k \sqrt{t}$. It should be noted that the data points of Fig. 2 (upper) did not fall on a straight line when plotted on semilog axes. In other words, buildup was not exponential.

The buildup of the classical movement aftereffect has been reported to be linear up to about $60 \mathrm{sec}$. 
saturating for longer durations (Lehmkuhle and Fox, 1975). Thus it seems clear that our depth movement aftereffect builds up with a different timecourse and over about 10 times longer duration than the classical movement aftereffect.

Figure 2 (lower), plotted on semilog axes, shows that the aftereffect decayed exponentially and obeyed the equation $V_{N}=15.1 \exp (-t / 52)$ where $V_{N}$ is the rate of change of size (in $\mathrm{min}$ arc/sec) required to null the aftereffect $t$ seconds following the cessation of $20 \mathrm{~min}$ adaptation. The decay time constant was therefore about $52 \mathrm{sec}$ (time taken to decay to $\mathrm{e}^{-1}$ of the initial value). We should note that the data of Fig. 2 (lower), clearly did not fit a straight line when plotted on $\log -\log$ axes so that, in contrast to the buildup of the aftereffect. the decay did not follow a power law. It is interesting to note that although the classical motion aftereffect also decays with an exponential time course (Honig, 1967) the time constant is very much shorter (10-30 times).

\section{Interocular transfer}

In order to find whether any part of our aftereffect was due to adaptation of a binocularly-driven neural mechanism. we measured the interocular transfer of the effect. Firstly, the right eye was adapted for $20 \mathrm{~min}$ to increasing size. During this period the left eye viewed a background of equal luminance to that viewed by the right eye. Then the head was rapidly moved to enable the left eye to view the stimulus, and the aftereffect nulled. The value of nulling rate of size change for the left eye was $42 \%$ that of the right for subject K.B. and $37 \%$ for subject D.R. Subject K.B.'s right eye was weakly dominant and D.R.'s was strongly dominant. Since binocularly-driven neurons are not found in the geniculocalcarine pathway at pre-cortical level, this result is consistent with a site either in cortex or colliculus for the neural mechanism sensitive to changing size.

\section{Retinal localization}

The aftereffect disappeared when the point of fixation was shifted through about half a side's length from the centre of the static test square. The transferred aftereffect was similarly localized. Thus, this aftereffect seems to be restricted to a small region of the visual field not much more extensive than the adapting square. Furthermore, when the point of gaze was suddenly shifted during adaptation from the centre of the square to outside the square, the size changes appeared to grow much larger, an effect not obvious before adaptation. In other words, sensory magnitude diminished during adaptation and this effect was also localized.

\section{Positive and negative contrasts}

Finally, we asked whether the aftereffect was sensitive to the sign of spatial contrast. The stimulus was either a bright square or a dark square. The motionin-depth aftereffect generated by the bright square was clearly evident with either square as the test stimulus. Furthermore, the illusory motion following adaptation to the bright square was directed away from the subject for either test square when the positive adapting square increased in size, and the aftereffect could be nulled by some unique rate of size increase for either test square. Again, illusory motion following adaptation to the negative-contrast square was away from the subject for either test square when the negative adapting square increased in size and the aftereffect could be nulled by some unique rate of size increase for either test square. Adaptation to decreasing size produced an aftereffect similarly indifferent to the sign of contrast (test or adapting). The aftereffect caused by either adapting square showed interocular transfer for both test squares. The percentage of interocular transfer depended on the stimulus conditions (i.e. it differed for increasing-decreasing size, positive-negative contrast of the adapting square and positive-negative contrast of the test square).

\section{DISCUSSION}

In separate experiments we found the following evidence for our proposal that neural filters sensitive to dynamically changing size exist in the human visual pathway (Regan and Beverley, 1978). We adapted to a square whose edges oscillated sinusoidally. When opposite edges oscillated in phase, the whole square moved from side-to-side, without changing size: when opposite edges oscillated $180^{\circ}$ out of phase, the square's size oscillated. Note that movements of any given edge were identical in the two conditions. We adapted to (a) oscillatory size and (b) oscillatory motion, then tested with (c) oscillatory size and (d) oscillatory motion. We found a large depression of visual sensitivity specific to "adapt size-test size" [i.e. (a)-(c)]: there was no comparable effect for (a)-(d), (b)-(c) or (b)-(d). In other words, we found adaptation specific to the relationship between movements of opposite edges. This discounts explanations in terms of the classical motion aftereffect. It locates the adaptation at or central to the stage where signals from opposite edges come together, signalling motion in opposite directions. In other words, adaptation occurs at or central to a stage sensitive to changing. size. On this basis (plus the results of certain control experiments) we proposed that information as to dynamically changing size is processed in separate information-processing channels, and suggested that these might correspond to the existence within the human visual pathway of neural organizations (or single neurons) preferentially sensitive to changing size (Regan and Beverley, 1978). The activity of this neural mechanism might partly account for the precise judgements of the direction of motion in depth made by one-eyed gamesplayers and jet pilots (Lewis and Krier, 1969; Lewis, Blakeley, Swaroop, Masters and McMurty, 1973).

We now report that adaptation to ramping changes of size generates a negative aftereffect (viz. illusory motion in depth) that can be nulled by a real stimulus (dynamically changing size). This further supports our previous proposal that changing-size filters exist.

There is a qualitative difference between our aftereffect and the adapting stimulus that generates it. This is an important distinction from previously-known aftereffects such as, for example, the Waterfall effect and the tilt aftereffect since it implies that the motionin-depth aftereffect cannot be explained along the lines proposed by Sutherland (1961) and Barlow and 
Hill (1963). In order to account for the observation that our aftereffect was of movement in depth rather than changing size we propose that the outputs of the changing-size filters can drive the neural organization that underlies the perception of motion in depth. Evidence against more parsimonious explanations based on the classical motion aftereffect is that our aftereffect builds up and decays over durations completely different from the buildup and decay of the classical aftereffect.

At the single-neuron level, several explanations for our findings are ruled out by the point that the motion-in-depth aftereffect's direction is determined by the direction of size change independently of whether contrast is positive or negative. We have argued elsewhere that the operation of our changing size filters are (to a first approximation) indifferent to the sign of stimulus contrast (Regan and Beverley, 1978). This would be sufficient to explain our present findings since visual information passing from the changing-size filters to the neural organization that underlies the perception of motion-in-depth would then be independent of whether stimulus contrast were positive or negative.

One question that springs to mind is what relation there might be between our aftereffect and the wellknown observation that after gazing at a rotating spiral, a stationary spiral appears to be moving radially inwards or outwards. Like our effect the spiral aftereffect shows interocular transfer, and is restricted to a local area of the visual field in both the adapted eye and the non-adapted eye (Walls 1953). The spiral aftereffect is often cited as demonstrating the aftereffect of linear motion (Waterfall Effect) (Kling and Riggs, 1972). Evidence reported here and elsewhere (Regan and Beverley, 1978), however, make it improbable that our effect is caused by the well-known adaptation of linear motion detectors (Wohlgemuth, 1911; Pantle and Sekuler, 1968). It seems likely that future explanations of the spiral aftereffect may have to consider not only adaptation of motion detectors but also adaptation of detectors sensitive to changing size.
Acknowledgements-We thank Drs W. K. Honig. B. Timney and $\mathrm{M}$. Cynader for their comments on this $\mathrm{Ms}$. W' are grateful to Nancy Beattie for assistance in preparing this manuscript. We thank the Canadian NRC for grant support. K.I.B. held a Killam Postdoctoral Fellowship.

\section{REFERENCES}

Barlow H. B. and Hill R. M. (1963) Evidence for a physiological explanation of the waterfall phenomenon and figural aftereffects. Vature, Lond. 200. 1345-1347.

Beverley K. I. and Regan D. (1973a) Selective adaptation in stereoscopic depth perception. J. Physiol, Lond. 232. $40-41 \mathrm{P}$.

Beverley K. I. and Regan D. (1973b) Evidence for the existence of neural mechanisms selectively sensitive to the direction of movement in space. J. Physiol. Lond. 235. 17-29.

Beverley K. I. and Regan D. (1975) The relation between discrimination and sensitivity in the perception of motion in depth. J. Physiol., Lond. 249. 387-398.

Cynader M. and Regan D. (1978) Neurons in cat parastriate cortex sensitive to the direction of motion in three-dimensional space. J. Physiol. Lond. In press.

Honig W. K. (1967) Studies of the "storage" of the aftereffect of seen movement. Abstract. Psychonomic Soc.

Kling J. W. and Riggs L. A. (1972) Woodworth and Schlos. berg's Experimental Psychology. Methuen, London.

Lehmkuhle S. W. and Fox R. (1975) Effect of binocula rivalry suppression on the motion aftereffect. V'ision Res. 15. 855-859.

Lewis C. E. Jr. and Krier G. E. (1969) Landing performance in jet aircraft after the loss of binocular vision. Aerospace Med. 40, 90-112.

Lewis C. E. Jr., Blakeley W. R., Swaroop R., Masters R. S. and McMurty T. O. (1973) Landing performance by low time private pilots after the sudden loss of binocular vision. Aerospace Med. 44, 1241-1245.

Pantle A. and Sekuler R. W. (1968) Velocity-sensitive elements in human vision: initial psychophysical evidence Vision Res. 8, 445-450.

Regan D. and Beverley K. I. (1978) Looming detectors in the human visual pathway. In preparation.

Sutheriand N. S. (1961) Figural aftereffects and apparent size. Q. J. exp. Psychol. 13, 222-228.

Walls G. L. (1953) Interocular transfer of after images. Am. J. optom; Archs Am. Acad. optom. 30, 57-60.

Wohlgemuth $A$. (1911) On the after-effect of seen movement. Br. J. Psychol. Suppl. I. 